Math. Model. Nat. Phenom.

Vol. 2, No. 4, 2007, pp. 63-73

\title{
Seasonal forcing drives spatio-temporal pattern formation in rabies epidemics
}

\author{
N. v. Festenberg ${ }^{a 1}$, T. Gross $^{b}$ and B. Blasius ${ }^{c}$ \\ ${ }^{a}$ Lehrstuhl Computergraphik und Visualisierung, Technische Universität Dresden \\ Dresden, Germany \\ ${ }^{b}$ Department of Chemical Engineering, Princeton University \\ Princeton, USA \\ ${ }^{c}$ Institut für Chemie und Biologie des Meeres, Universität Oldenburg \\ Oldenburg, Germany
}

\begin{abstract}
Seasonal forcing is identified as a key pattern generating mechanism in an epidemic model of rabies dispersal. We reduce an established individual-based high-detail model down to a deterministic conceptual model. The characteristic wave pattern characterized by high densities of infected individuals is maintained throughout the reduction process. In our model it is evident that seasonal forcing is the dominant factor that drives pattern formation. In particular we show that seasonal forcing can cause splitting of epidemic wave fronts.
\end{abstract}

Key words: pattern formation, epidemics, rabies, seasonal forcing

AMS subject classification: 65P40, 65C99, 92P99

\section{Introduction}

Today, infectious diseases are becoming an ever greater threat to humankind. One factor adding to this growing threat is that we are changing the environment at an accelerating pace. As we alter natural habitats we face new disease vectors and encounter known ones in new settings [13]. Therefore, we witness the emergence of new diseases, such as HIV (1981) and SARS (2002) [18, 19, 7], and the reappearance of established ones, e.g. tuberculosis. The red fox (Vulpes vulpes), the most important vector for rabies in Europe, is more and

\footnotetext{
${ }^{1}$ Corresponding author. E-mail: nf6@inf.tu-dresden.de
} 
more often encountered in urban areas. This increases the risk of rabies transmission to humans.

Rabies is an infectious disease of the central nervous system. Once the disease has reached clinical stage in humans mortality is almost $100 \%[16,9]$. Because of the importance of the red fox as a vector, the epidemiology of rabies is invariably linked to the foxes' ecology. The spreading of rabies in Europe is characterized by the appearance of epidemic wave fronts. A peculiar feature is that, behind the first wave of infections in a new area, subsequent smaller waves follow $[1,15,12,16]$.

In order to combat epidemics, such as rabies, detailed knowledge of the spreading of the disease can greatly enhance the effectiveness of vaccination campaigns $[20,4]$. For this purpose the investigation of mathematical models can provide valuable insights. To forecast the spreading of a specific disease realistic models that describe the disease in great detail are needed. Nevertheless, more abstract conceptual models have to be considered to understand general mechanisms of epidemic spreading.

Modelling approaches to epidemics date back to [3] in the eighteenth century. The foundation of modern epidemiological modelling was laid by Kermack and McKendrick [14], who introduced the concept that a population can be subdivided into several classes. Each of these consists of individuals in a specific epidemiological state.

Many phenomena in epidemics, such as epidemic wave fronts in rabies, are intrinsic spatio-temporal effects $[10,21,2]$. The investigation of these phenomena requires spatial models. In particular in recent years explicit individual-based models have received much attention $[5,8]$. An inherent advantage of these models is that they capture the discrete nature of the individual animals.

An influential model for rabies spreading was proposed in [11]. This model is based on the well-known paradigm of a cellular automaton (see [6, 22]). It incorporates many details of the foxes' ecology. This model is valuable as it shows that realistic individual-based interactions can give rise to the observed epidemic waves including secondary wave fronts behind the initial front. Jeltsch et al. argue that rare long range interaction events due to migrating young foxes are the key pattern generating mechanism. However, the small-scale focus of individual-based models makes it very difficult to investigate system-level phenomena with certainty.

In this paper we systematically reduce the full rabies model of Jeltsch et al. to extract the pattern generating mechanism. This is in contrast to [26] who attempt a similar analysis by starting from a simple model with more and more details added subsequently. Our analysis reveals that migration of foxes is of minor importance compared to the seasonal forcing of the model. We propose a deterministic conceptual model that reproduces the observed wave pattern. In addition this model shows that seasonal forcing can lead to the splitting of epidemic wave fronts. We argue that this wave splitting mechanism is the cause of the observed secondary waves. Wave splitting means here that a single wave front travelling into one direction is transformed into a pair of wave fronts travelling in opposite directions.

We start in Section 2 by describing the full rabies model used in our analysis. In Section 3 we present all our reduction steps. In Section 4 we propose the mechanism of wave splitting 
as explanation for the observed wave patterns. Section 5 concludes with a suggestion to potential use of our results.

\section{Modelling approaches to rabies}

In this section we discuss different modelling approaches to rabies. A central feature a credible model has to reproduce is the emergence of secondary waves behind the primary front. The emergence of secondary waves is a suitable measure to assess a model's quality, because it indicates a system's ability to produce spatiotemporal oscillations - a phenomenon regularly observed in rabies outbreaks.

Many models discussed in the literature are adaptations of the Kermack-McKendrick approach, e.g. $[1,15,16,25]$. Despite differences in details all of these models are variants of spatial SIR models. In such models the local densities of susceptible, infected and recovered individuals are continuous variables. While these models exhibit wave patterns in infection densities, the mechanism that causes secondary waves is not clear in comparison with the real world. In a continuous model the local densities of infected do generally not exactly become zero. Even after the primary infection has swept through an area, a small residual density of infected remains. These infected can then trigger a secondary wave. Although this mechanism produces secondary waves in models it is doubtful that it offers a good description of the situation in nature, since the low residual densities of infected can often be lower than a single individual. A way to avoid this problem is to resort to individualbased models. A convenient framework for such models are cellular automata $[10,6,12]$. A cellular automaton consists of a set of $N$ cells which are organized on a grid so that all cells have a local neighbourhood $U$. Each cell has a discrete state that is updated according to a set of possibly stochastic rules.

A comprehensive rabies model based on a cellular automaton is proposed in [11]. Since fox families live in close contact, a family rather than an individual constitutes the basic unit of the model. Each cell represents a potential home range for a single fox family. The cell can be either populated and susceptible $(S)$, populated and infected $(I)$, or empty ( $R$ for removed). The neighbourhood in the model consists of the eight nearest cells. The update is performed synchronously [22] with stochastic rules. The model describes neighbourhood infections, recovery, death and repopulation by migration. In particular migration of foxes is modelled in great detail relying on empirical data [27, 24]. Once a year (every sixth time step) young foxes leave their home ranges either repopulating empty ranges or merging with populated ranges. Each migrating fox is represented individually. Both infected and susceptible young foxes start their migration towards a random main direction. In each migration step they choose to proceed along the main direction or with equal probability they take a step one range to the left or to the right. With a certain probability the foxes stop the migration and stay in the range which they traverse. This probability increases linearly with the distance from their home range. It also depends on the state of the traversed cell. For empty cells the staying probability is higher and increases faster than for healthy or infected cells. With the parameter values used in the model this leads to a probability 
distribution for the migration distance, with a maximum distance around four ranges and an exponential tail of up to 60 ranges for non-empty target ranges. Both traversed and target ranges can be infected, the latter with a higher probability. Note that these rules introduce a long range interaction between cells. In the following we refer to the model described here as the full rabies model. For further details refer to [11].

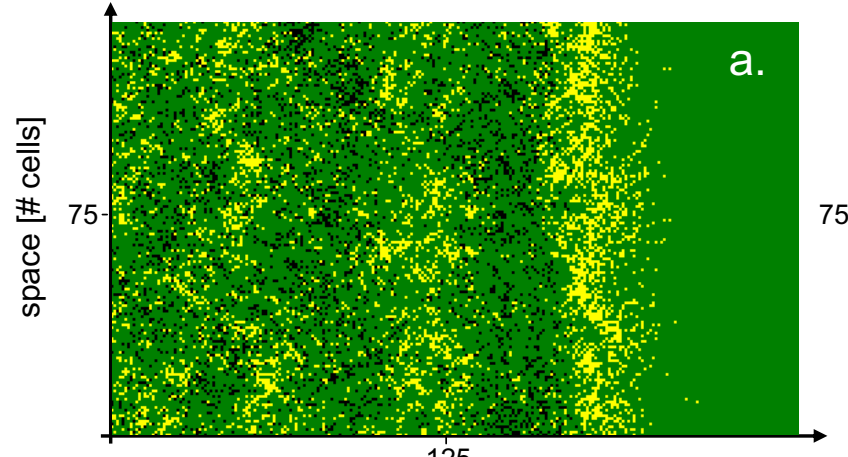

125

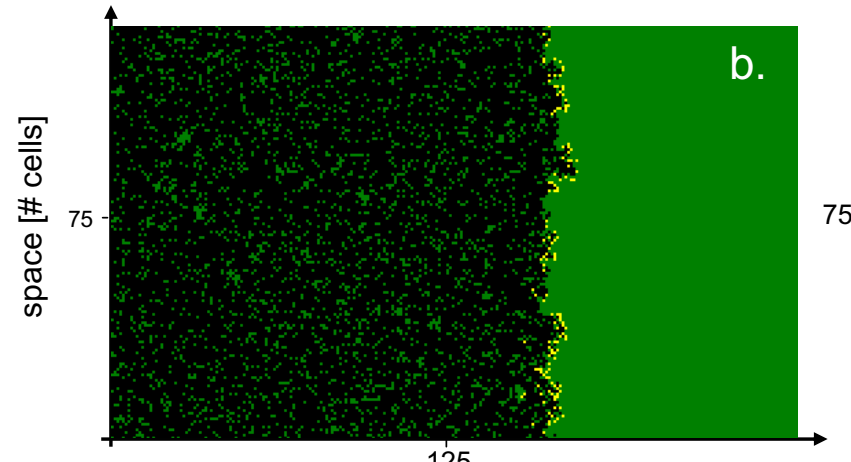

125

space [\# cells]
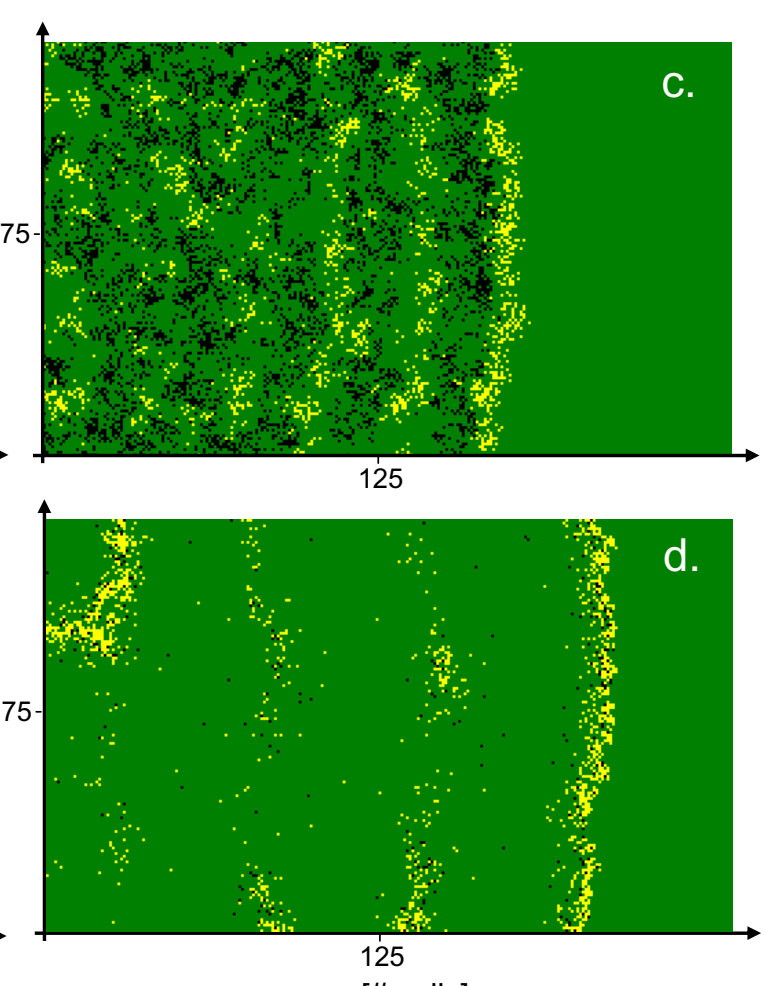

space [\# cells]

Figure 1: Typical wave patterns observed a.) in the full rabies model [11], b.) the full model with the long range rule switched off, c.) the full model with the maximum distance of migration set to two; d.) Analogous SIR model with temporally forced repopulation rate ( $T=40$, see Fig. 2 for details). Green denotes susceptible, yellow infected and black empty or removed cells. The initial condition in all pictures was a line of infected at the left border in an otherwise susceptible population. The spatio-temporal dynamics in the full model is characterized by recurrent wave fronts (a). These fronts can also be observed in simplified models $(\mathrm{c}, \mathrm{d})$. Without long range interaction only the primary wave front can be observed as empty ranges can not be repopulated. The area shown consists of $250 \times 150$ cells.

In Fig. 1a a typical snapshot from the full model is shown. The primary, secondary and tertiary fronts can be spotted in the figure. Barely any empty cells are visible because the repopulation rate is high and the picture is taken after a repopulation event. 


\section{Reduction to a conceptual model of rabies spreading}

While the full model reproduces the secondary waves, which are observed in field data, it contains many details that complicate the analysis. In the following we reduce the model to a conceptual simplified form.

Leaving out the details of the migration process the transitions in the full rabies model can be formulated as

$\begin{array}{ccc}S & \stackrel{p_{1}(U), p_{2}^{\prime}, p_{3}^{\prime}}{\longrightarrow} & I \\ I & \stackrel{q_{1}}{\longrightarrow} & R \\ I & \stackrel{q_{2}}{\longrightarrow} & S \\ R & \stackrel{r^{\prime}}{\longrightarrow} & S .\end{array}$

where $p(U)$ is the infection probability depending on the local neighbourhood $U, p_{2}^{\prime}$ is the infection probability from infected migrating foxes traversing a range, $p_{3}^{\prime}$ is the infection probability from infected migrating foxes staying in a range, $q_{1}$ and $q_{2}$ is the mortality and recovery rate (with $q_{1}+q_{2} \leq 1$ and $q_{1}>q_{2}$ ) and $r^{\prime}$ is the repopulation rate. Here all processes involving long range interactions have been marked with a prime.

The model's crucial feature is the seasonal migration of young foxes which repopulates empty cells and enhances the spreading of the epidemic. This migration introduces both long-range interaction and strong seasonal forcing. In [11] it is argued that rare long-range dispersal causes the observed secondary waves. Our analysis confirms that migration is indeed necessary for the emergence of these waves. However, we show that it is the temporal forcing rather than the non-local nature of the interaction that causes the observed wave pattern.

In order to understand the pattern generating mechanism further simplifications are necessary. To derive a simpler model we have used a stepwise trial-and-error strategy, in which details of the model were removed, while we tried to maintain the wave pattern. In this way a mathematically simple model can be found.

In the first reduction step we ignore all interactions along a migrating fox's trajectory, except at the end point of the migration. While the trajectory itself is still computed according to the individual-based rules, only the location of the end point is of importance. This leaves the observed wave pattern effectively unchanged.

As a second step we vary the long range interaction. By setting $p_{2}^{\prime}, p_{3}^{\prime}$ and $r^{\prime}$ to zero we can switch migration off completely. In this case emptied regions can not be repopulated. The first epidemic front that passes through the system leaves behind almost emptied space. In this space the density of foxes is too low to sustain secondary waves (see also [17]). This situation is shown in Fig. 1b.

In the following we set the maximum distance a fox can travel to two. This setting allows for repopulation of emptied areas while reducing the range of interaction to a minimum. For comparison, a value of 60 is used in [11]. The emerging wave pattern is shown in Fig. 1c. Reducing the migration distance leads to a reduced dispersal speed. It also has a small 
impact on the wave length of the observed pattern. However, the effect on wavelength is relatively weak. In particular no further decrease in wavelength occurs below a distance of ten. Even at a parameter value of two, the wave pattern remains qualitatively similar to the pattern observed at 60 .

The results described above show that the non-local nature of the long range interaction is not the mechanism that drives pattern formation. As we have seen, the importance of migration mainly lies in the repopulation of emptied cells. It is a crucial feature of the full model that this repopulation takes place seasonally. We can mimic these dynamics in a much simpler framework, by considering a model without long range interactions.

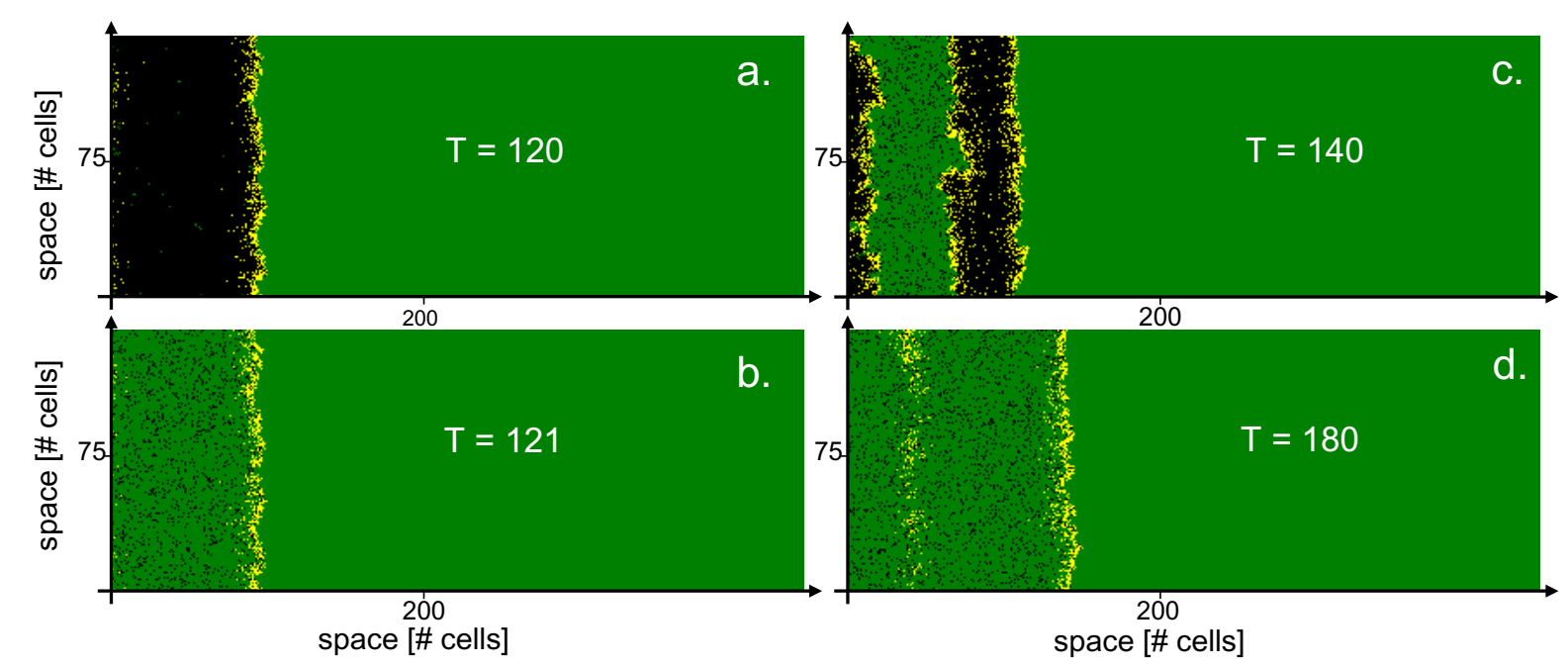

Figure 2: Results from the individual-based SIR model after a.) 120, b.) 121, c.) 140, and d.) 181 steps. Temporal forcing that enters through the seasonal repopulation of empty ranges can cause splitting of epidemic wave fronts. With: $p=0.2, q_{1}=0.2, q_{2}=0.01$, $r(t)=r_{1} \delta(t \cdot \bmod T)+r_{0}$ with $T=60$ and $r_{1}=0.9, r_{1}=0,400 \times 150$ cell, initial conditions as in Fig. 1

Emanating from Eq. (3.1) we set $p_{2}^{\prime}=p_{3}^{\prime}=0$ and replace $r^{\prime}$ by a time-dependent, neighbourhood-independent function

$$
r(t)=r_{1} \delta(t \cdot \bmod T)+r_{0}
$$

where $\delta()$ is the $\delta$-distribution (being non zero for arguments equal to zero only) and mod denotes the modulo operator. The parameters are set to $r_{1}=0.9$ and $r_{0}=0$ which results in a realistic repopulation rate. This repopulation rule is more abstract than the one used in the full model, but empirically consistent with the observation that almost all empty ranges are repopulated once a year. Instead of having several foxes traversing a home range and eventually staying there this rule simply assumes that a range is repopulated with a high probability. What matters here is that $r(t)$ is much higher than $50 \%$ every $T$ th step. The 
model effectively is an individual-based spatial SIR-model with temporal forcing. As the full model this conceptual model exhibits a pattern of recurrent waves. Note that the long range interaction is completely removed now. A representative snapshot is shown in Fig. 1d.

\section{Wave splitting of epidemic wave fronts}

In this simple model another phenomenon can be observed. The strong temporal forcing included in the model can lead to a splitting of epidemic wave fronts. In Fig. 2 a sequence of the propagating epidemic front in the reduced $2 \mathrm{~d}$-model is shown. Typically, epidemic waves propagate in one direction, since the emptied area behind the wave can no longer sustain the epidemic. However, in a repopulation event the emptied area behind the wave is filled. Subsequently the wave can split into two waves, propagating in opposite directions.

Initially, the repopulation events double the number of wave fronts. Since the total area that is accessible to the epidemic is limited, collisions of wave fronts have to occur. In principle one can imagine that two counter-propagating wave fronts annihilate each other in a collision. At the time, at which both waves meet, they are surrounded by emptied area on both sides so that further propagation is impossible. But, because of the nature of the splitting, the next repopulation event has to occur at the time of collision (see Fig. 2d). Because of the new substrate of susceptible cells provided by the repopulation, the waves can survive the collision.

In order to understand the phenomenon of wave splitting in more detail we consider a model in one spatial dimension. In this model the local neighbourhood of a cell is set to the two nearest neighbours on each side, in order to obtain results comparable to the twodimensional model. As in the full model, a cell can be susceptible, infected or removed. Susceptible cells in the neighbourhood of an infected cell become infected with a probability $p$ in a given step. Infected cells are removed in each step with probability $q_{1}$. The seasonal repopulation event is modelled by turning all emptied cells into susceptible cells every 24th step.

Figure 3a shows a simulation run of the one-dimensional model. In this figure, one dimensional space is shown over a total of 1024 time steps. Since the two wave fronts from a splitting event propagate at the same speed $v$ they both cover a distance of $d=v T$ before the next repopulation event, where $T$ is the period of the forcing. As the next repopulation event occurs, both of these waves give rise to four second generation waves. Two of these second generation waves propagate towards each other. Both waves propagate at the speed $v$. Moreover, each wave covers the distance $d$ before collision. Therefore, the collision takes place at the location of the initial splitting event and occurs at $T+d / v=2 T$, that is, at the time of the second repopulation event. Note that the wavelength of the observed pattern is proportional to the repopulation period.

The synchrony of repopulation and wave collision is even more evident in the deterministic limit of the model. In this limit the transition probabilities of the one dimensional model are set to $p=q_{1}=1$. A simulation result of the deterministic model is shown in Fig. 3b. The space-time plot in the figure can be considered as the deterministic backbone of the pattern 
generating mechanism. In particular, the figure shows that repopulation and wave collision occur exactly at the same time in the deterministic limit. Let us emphasize that this is true independently of the parameters of the simulation.

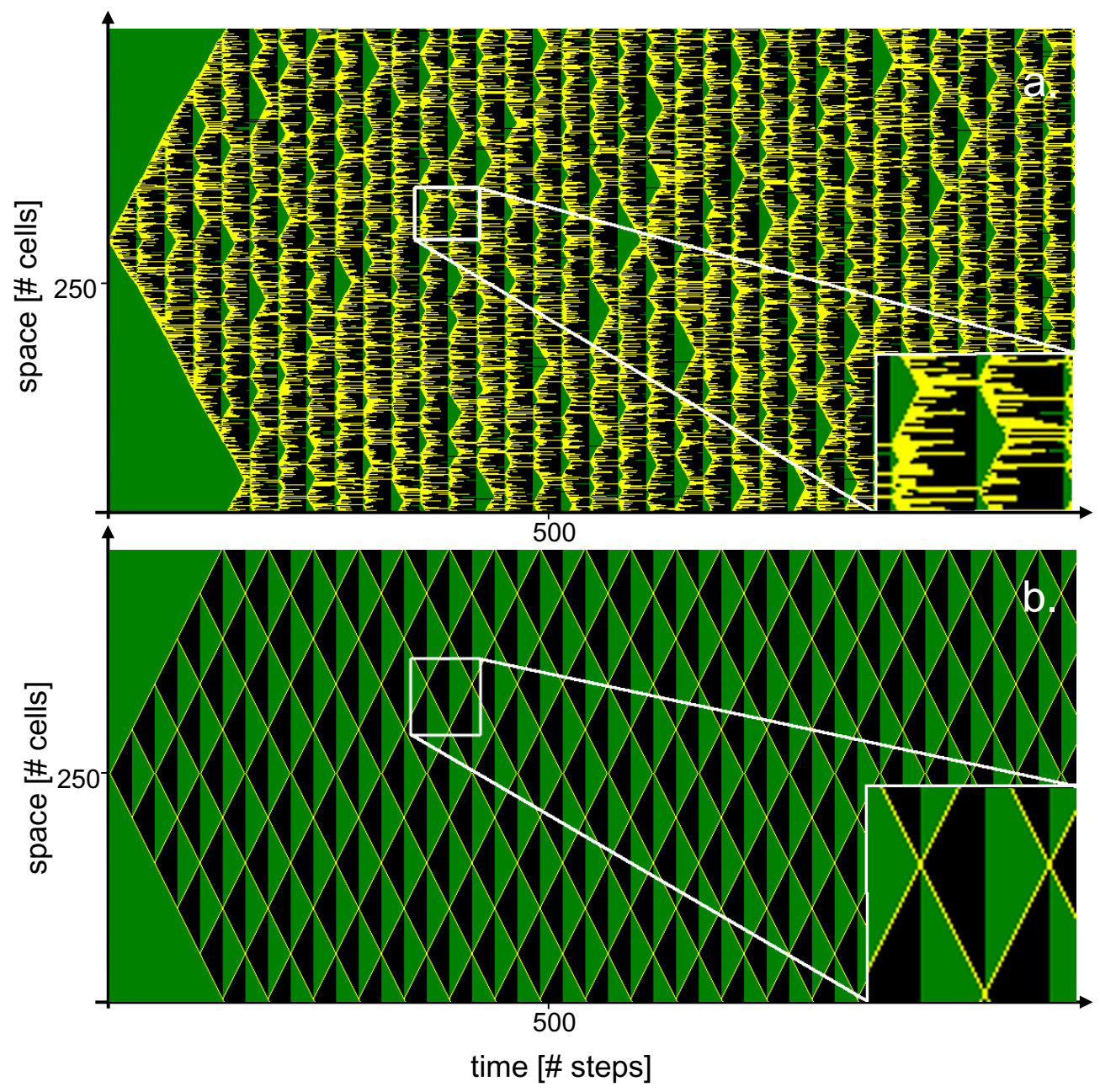

Figure 3: Spatio-temporal plot of the conceptual one-dimensional model (a) and its deterministic limit (b). Wave splitting leads to the generation of a set of epidemic wave fronts. Independently of the parameters the collisions of waves is likely to occur at the time of the repopulation events. Thereby the annihilation of waves can be avoided. Parameters: a.) $p=0.8, q_{1}=0.10, q_{2}=0, r_{0}=0.01, r_{1}=1$ and $T=24$ and b.) deterministic limit case $p=1, r_{0}=0, r_{1}=1, T=24$ and $q_{1}=1.1024$ time steps of 512 cells each. The inset shows the appearance of new wave sources in detail.

It is interesting to note that the mechanism described above generates waves that seem to originate from a set of discrete infection foci, e.g., the initial splitting site. Such foci are also observed in nature and are commonly attributed to spatial inhomogeneities. However, in our 
conceptual model, as well as in the full rabies model, foci emerge although the underlying space is homogeneous [11].

\section{Conclusion}

In this paper we showed that temporal forcing is the key mechanism that leads to the formation of recurrent epidemic wave fronts in an individual-based model of rabies spreading. By stepwise reduction of the model we derived a simple conceptual model exhibiting the same mechanism. While we started from a rabies model, the proposed conceptual model can describe epidemic waves in a larger class of epidemic diseases.

Our analysis reveals that the pattern formation mechanism here is not qualitatively different from reaction waves in conventional reaction-diffusion systems, such as SIR-models. In both cases new excited fronts emerge as soon as enough excitable medium is present again. In contrast to most other reaction-diffusion systems our model exhibits a pattern of periodic collision and splitting of epidemic wave fronts. This pattern arises by virtue of strong temporal forcing which enters through the seasonal reproduction cycle of the disease vectors.

Based on our analysis it appears possible that the secondary disease waves observed in nature originate from a wave splitting mechanism. In the literature such waves are often attributed to disease foci which arise because of spatial inhomogeneities. Although we observe similar foci in our simulation, they are formed by a self-organization of wave splitting events in the underlying homogeneous space.

Our findings emphasize the importance of migrating foxes for the emergence of epidemic wave fronts. This suggests that pulsed vaccination strategies, in step with the reproductive cycle can be effective [23].

Finally it needs to be stressed that both complex and conceptual models deliver valuable insights. Complex models such as in [11] striving for maximal realism allow for detailed understanding. However, in some cases as in ours, conceptual models offer more concise an access to the governing dynamical foundations of natural phenomena.

\section{References}

[1] R.M. Anderson, H.C. Jackson, R.M. May, A.M. Smith. Population dynamics of fox rabies in europe. Nature 289 (1981), 765-771.

[2] R.M. Anderson, R.M. May. Infectious diseases of Humans. Oxford University Press, Oxford, 1992.

[3] D. Bernoulli. Essai d'une nouvelle analyse de la mortalité causée par la petite vérole et des avantages de l'inoculation pour la prévenir. Mem. Math. Phys. Acad. Roy. Sci. Paris (1760), 1-45. 
[4] Z. Desz, A.-L. Barabaśi. Halting viruses in scale-free networks. Phys. Rev. E 65 (2002), 055103(R).

[5] R. Durrett, S. A. Levin. The importance of being discrete (and spatial). Theor. Popul. Biol. 46 (1994), 363-394.

[6] R. Durrett, S. A. Levin. Stochastic spatial models: a user's guide to ecological applications. Phil. Trans. R. Soc. Lond. B 343 (1994), 329-350.

[7] M. Enserink, G. Vogel. Infectious diseases - deferring competition, global net closes in on sars. Science 300 (2003), 224-225.

[8] T. Gross, C.J. Dommar, B. Blasius. Bistability and oscillations in a sis model on an adaptive network. Phys. Rev. Lett. 96 (2006), 208701.

[9] K. Holmala, K. Kauhala. Ecology of wildlife rabies in europe. Mammal Review 36 (2006), 17-36.

[10] E.H. Holmes. Basic epidemiological concepts in a spatial context. In: Spatial Ecology (D. Tilman, ed.). Monographs in Pop. Biol., Vol. 30. Princeton University Press, Princeton, 1997.

[11] F. Jeltsch, M.S. Müller, V. Grimm, C. Wissel, R. Brandl. Pattern formation triggered by rare events: lessons from the spread of rabies. Proc. R. Soc. Lond. B 264 (1997), 495-503.

[12] A. Johansen. A simple model of recurrent epidemics. J. theor. Biol. 178 (1996), 45-51.

[13] A. Karlen. Man and microbes: Disease and plagues in history and modern times. Touchstone, 1996.

[14] W. O. Kermack, A. G. McKendrick. A contribution to the mathematical theory of epidemics. Proc. R. Soc. Lond. A 115 (1927), 700-721.

[15] A. Kallén, P. Arcuri, J.D. Murray. A simple model for the spatial spread and control of rabies. J. theor. Biol. 116 (1985), 377-393.

[16] J. D. Murray. Mathematical Biology, Vol. 1 \& 2. Springer, New York, 2002.

[17] M.E.J. Newman. Threshold effects for two pathogens spreading on a network. Phys. Rev. Lett. 95(2005), 108701.

[18] M.B.A. Oldstone. Viruses, plagues and history. Oxford University Press, Oxford, 1998.

[19] R. Olinky. Threshold in epidemiological models. PhD thesis. Tel Aviv University, 2005.

[20] R. Pastor-Satorrasi, A. Vespignani. Immunization of complex networks. Phys. Rev. E 65 (2002), 036104. 
[21] P. Rohani, D.J.D. Earn, B.T. Grenfell. Opposite patterns of synchrony in sympatric disease metapopulations. Science 286 (1999), 968-971.

[22] B. Schönfisch, A. de Roos. Synchronous and asynchronous updating in cellular automata. BioSystems 51 (1999), 123-143.

[23] B. Shulgin, L. Stone, Z. Agur. Pulse vaccination strategy in the sir epidemic model. Bull. Math. Biol. 60 (1998), 1123-1148.

[24] F. Steck, A. Wandeler. The epidemiology of fox rabies in europe. Epidemiol. Rev. 2 (1980), 72-96.

[25] C. Suppo, J.-M. Naulin, M. Langlais, M. Artois. A modelling approach to vaccination and contraception programmes for rabies control in fox population. Proc. R. Soc. Lond. B 276(2000), 1575-1582.

[26] H.-H. Thulke, V. Grimm, M.S. Müller, C. Staubach, L. Tischendorf, C. Wissel, F. Jeltsch. From pattern to practice: a scaling down strategy for spatially explicit modelling illustrated by the spread and control of rabies. Ecol. Mod. 117 (1999), 179-202.

[27] B. Toma and L. Andral. Epidemiology of fox rabies. Adv. Virus Res. 21 (1977), 1-36. 